\title{
Vortex arms - symmetric vortices in the wake of an oscillating cylinder
}

Pieter Boersma ${ }^{1}$, Erica DeWitt ${ }^{2}$, Cami Quinteros ${ }^{2}$, Fey Thurber ${ }^{2}$, Tyler Gurian ${ }^{1}$, Pari Riahi², Yahya Modarres-Sadeghi ${ }^{1}$

${ }^{1}$ Department of Mechanical and Industrial Engineering, University of Massachusetts Amherst, Amherst, MA 01002, USA

${ }^{2}$ Architecture Department, University of Massachusetts Amherst, Amherst, MA 01002, USA
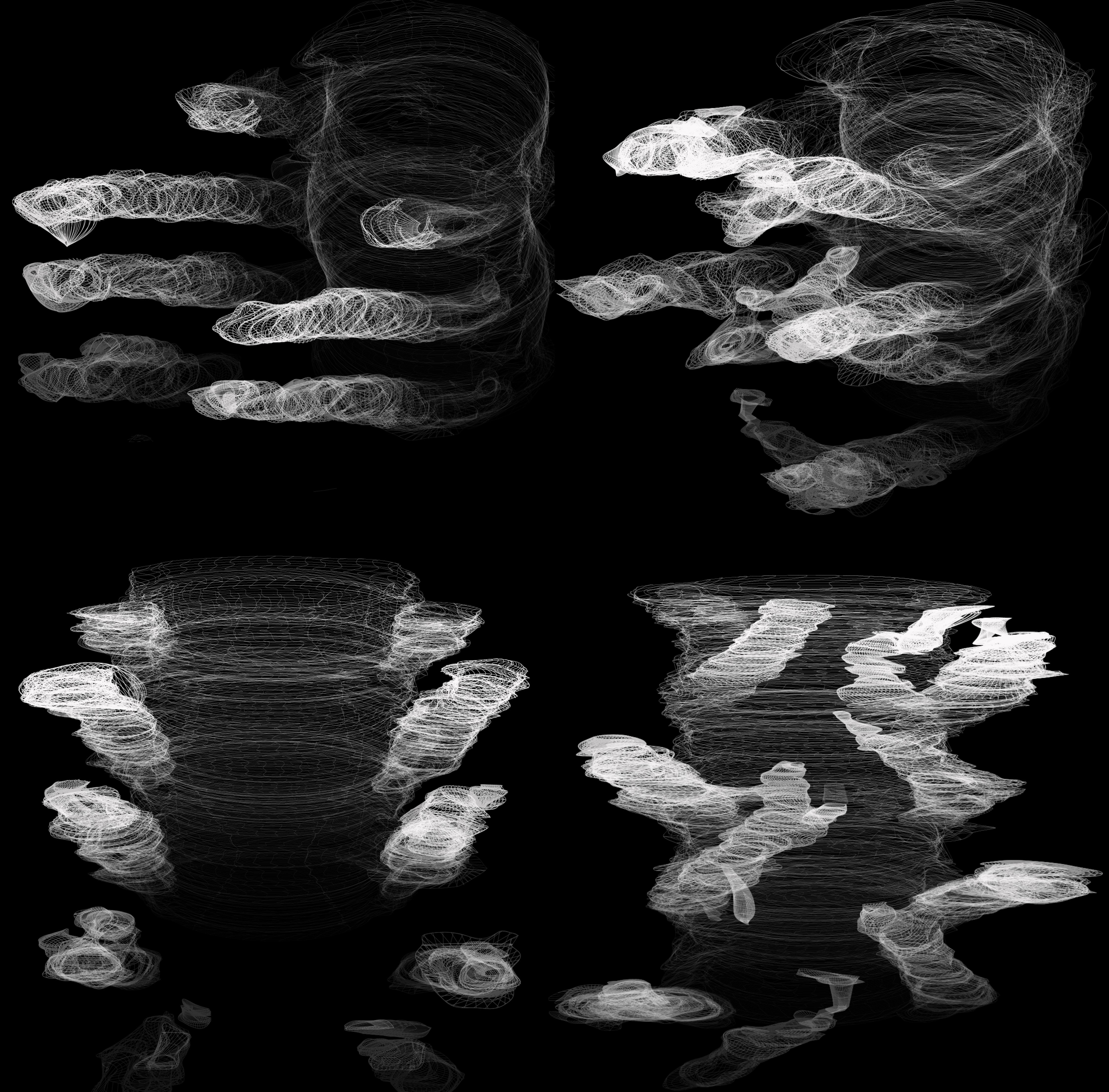

Symmetric vortices are shed in the wake of a cylinder that is free to oscillate in the direction of flow. At lower flow velocities (left) these vortices stay parallel to each other in the wake. At slightly higher flow velocities (right) the vortices are shed simultaneously, but their size and direction of motion in the wake alternate in each cycle of oscillations. We call these vortices alternating symmetric vortices. 